Veritas Et Scientia

Vol. 7, N 2, 953-962

Julio - Diciembre del 2018.

ISSN Edición Online: 2617-0639

https://doi.org/10.47796/ves.v7i2.72

\title{
VALIDACIÓN DE UN INSTRUMENTO SOBRE MOTIVACIÓN Y ACTITUD POR EL APRENDIZAJE DE LA ODONTOLOGÍA LEGAL Y FORENSE EN ESTUDIANTES DEL IX CICLO DE LA ESCUELA DE ODONTOLOGÍA DE LA UNIVERSIDAD PRIVADA DE TACNA. 2017.
}

\author{
VALIDATION OF AN INSTRUMENT ON MOTIVATION AND ATTITUDE FOR THE LEARNING \\ OF LEGAL AND FORENSIC DENTISTRY IN STUDENTS OF THE NINTH CYCLE OF THE \\ SCHOOL OF DENTISTRY OF THE PRIVATE UNIVERSITY OF TACNA. 2017.
}

José Elard Núñez Cárdenas ${ }^{1}$

Presentado: $24 / 03 / 2018$

Aceptado: $23 / 04 / 2018$

Publicado online:27/12/2018

\section{RESUMEN}

Con este trabajo se menciona el proceso de elaboración de un instrumento que fuera validado para medir la motivación y las actitudes de los estudiantes del curso de pregrado Odontología Legal y Forense que se dicta en la Escuela Profesional De Odontología. Se aplicó el instrumento en grupo experimental de 46 estudiantes. El instrumento tiene dos partes, la primera es un test de motivación y la segunda parte un test de actitud ambos bajo la modalidad Likert. El instrumento fue ajustado por juicio de expertos y a través del contraste de confiabilidad y consistencia de alfa de Cronbach, alcanzando un valor de 0.8 . Con este análisis se confirma que el cuestionario es congruente y prueba por confiabilidad interna sus consistencias, para aplicarse a estudiantes del curso de Odontología Forense además que puede adaptarse a otras asignaturas.

Palabras clave: Motivación, actitud, odontología legal y forenseL

\section{ABSTRACT}

\footnotetext{
1 Magíster en Docencia Universitaria Y Gestión Educativa
} 
This work mentions the process of developing an instrument that was validated to measure the motivation and attitudes of students in the undergraduate course of Legal and Forensic Dentistry that is taught in the Professional School of Dentistry. The instrument was applied in an experimental group of 46 students. The instrument has two parts, the first is a motivation test and the second part an attitude test both under the Likert mode. The instrument was adjusted by expert judgment and by contrasting reliability and consistency of Cronbach's alpha, reaching a value of 0.8 . With this analysis, it is confirmed that the questionnaire is congruent and tests its consistencies for internal reliability, to be applied and adapted to other subjects.

Keywords: Motivation, Attitude, Legal and Forensic Dentistry.

\section{INTRODUCCIÓN}

La actitud y la motivación son aspectos psicológicos que se consideran importantes en el campo académico, Así por ejemplo en el ámbito educativo es importante valorar las reacciones afectivas que pueda mostrar el estudiante, por ejemplo, al estudio de las ciencias naturales, sociales, etc.; ya que de esa actitud, motivación o creencia dependerá el desarrollo personal y profesional del estudiante. La actitud que asuma el estudiante, y la motivación que mueva u oriente al estudiante hacia el estudio de la odontología legal forense podría darnos a conocer o a entender cómo enfocar las estrategias de enseñanza aprendizaje de este campo de estudio. La forma en que los estudiantes adquieren conocimientos o aprenden es variada, y las actitudes y motivaciones no son innatas, sino más bien adquiridas, así podemos decir, que existe una predisposición, la cual puede ser negativa o positiva hacia algo. En nuestra revisión no hemos encontrado algún instrumento específico que mida las variables actitud y motivación orientado al estudio de la odontología legal y forense, tomando en cuenta que si queremos intervenir en un determinado grupo de estudio es necesario contar con instrumentos de medición. Por lo que este trabajo se realizó con el objetivo de ofrecer un instrumento confiable y válido que mida las actitudes y motivaciones de los estudiantes del curso de odontología legal y forense.

La odontología forense es un curso de enseñanza que comprende el área de la clínica forense, donde la enseñanza aprendizaje está orientada principalmente a la identificación de personas o cuerpos desconocidos, además de estimar características de la edad o los tipos de lesiones; que como víctima haya sufrido, sea esta por accidentes, crímenes agresiones incluso llevándolos a la muerte, siendo este estudio de fragmentos de piezas dentarias o de intervenciones dentarias, además de la observación a los tejidos o mucosas bucales, cabeza y cuello. (INCIFO, 2017). Con respecto a las actitudes que se tiene en torno a la muerte dice Carrillo et. al. (Carrillo Esper, Contreras Carreto, Remolina Schlig, \& Ramírez Rosillo, 2007) que aún en estos tiempos paradójicamente el afrontamiento de la muerte es un tema de silenciamiento que lleva al familiar, pariente o personal profesional a una falta de encaramiento, ya que las emociones que traemos por vivencias culturales reprimen el modo de ver la cosas. Otro estudio (Madadin, 2013) muestra que las actitudes

Veritas et Scientia Vol. 7 (2) Julio - diciembre 2018. 
de los estudiantes de medicina hacia el curso de medicina forense en la Universidad de Dammam en Arabia Saudita eran pobres, el conocimiento inadecuado y la conciencia limitada para la importancia del curso en medicina forense. Por lo que vemos del estudio de Madadin, los estudiantes no consideran de gran importancia la información científica forense. Carstens Kõhler, Isabel Cristina. (2009) realiza un trabajo cualitativo para comprender la vivencia de los trabajadores del Instituto Médico Legal de Londrina. Sao Paulo Brasil; donde evalúa la representación del cadáver como objeto de trabajo y los sentimientos vividos por los trabajadores. El curso de odontología legal y forense implica el desarrollo de trabajos teóricos y prácticos vivenciales, donde el estudiante acude al Centro de Medicina Legal y es expuesto a las diversas circunstancias del día a día en la Morgue Central de Tacna, hasta el momento desconocemos cuál es la actitud. Mohammed S. Madadin, concluye en que se debe asumir la responsabilidad de aumentar la conciencia sobre el efecto de los medios de educación médica ofrece al estudiantado, y de lo que se debe incluir en el currículo del curso (Madadin, 2013). Vallejo, en Cuba afirma que las actitudes influyen en la calidad del aprendizaje y que se impone poner más atención al componente de la personalidad ante el fracaso. Galvez et al. llegaron a la conclusión lo necesario que resulta el estudio de la metodología forense. En el Perú se hizo un trabajo multicéntrico, donde Díaz Et. Al. encontraron que los estudiantes tenían actitudes positivas. Halló una relación positiva entre el nivel de actitud buena y pertenecer a un grupo de investigación, 2008). Rodríguez \& Loy expresa que Ciencia Forense es la aplicación de prácticas científicas dentro del proceso legal. (Rodríguez, 2016). El campo de acción de la ciencia forense es realmente amplio. Así, y de acuerdo al Reglamento del Instituto de Medicina Legal en el Perú en su Artículo 8 (Ministerio Público., 1998) las funciones generales del Instituto son regular las actividades de medicina legal en el país es dirigir, ejecutar, y evaluar el desarrollo de las actividades médico legales de su competencia, de acuerdo a las disposiciones legales en vigencia y a los lineamientos de política del Ministerio Público. Para su estudio se sabe que la motivación y las actitudes son variables afectivas que favorecen el aprendizaje e influyen en su perseverancia y en el deseo de interactuar(Minera Reyna, 2009). El estar o no motivado puede explicarnos el por qué unos tienen una alta producción mientras que otros no (Sandoval-Pineda, 2011). Donde "La motivación es un estado interno del individuo ...." (Minera Reyna, 2010). "un estado de disposición mental y nerviosa" (Ubillos, Mayordomo, \& Páez, 2015) y que implica metas que proporcionan impulso y dirección a la acción(Sandoval-Pineda, 2011) y en la medida que el hombre satisface sus necesidades surgen otras que cambian o modifican el comportamiento del mismo.(Quintero, J., 2012). Es el estado interno de la persona influenciado necesidades $y / o$ creencias que le generan actitudes e intereses favorables hacia una meta, (Minera Reyna, 2010). Se ha demostrado que la motivación influye positivamente en el rendimiento académico, y en el bienestar de los estudiantes. La teoría de la autodeterminación de la motivación considera que la calidad de la motivación es más importante que la cantidad (Kusurkar, 2013). Es así que en la toma de decisiones sobre el aprendizaje en el que el alumno se involucra estos aspectos. Es un proceso que no puede ser observado directamente, pero puede ser inferido por comportamientos como "elección de tareas, esfuerzo, persistencia y verbalizaciones" (Roberts, 2016). Los estudios de Lewin, demuestran que el poder de desarrollar empatía, no es un don innato en el hombre, sino una actitud adquirida y desarrollada por el aprendizaje (Barboza,1987).

Veritas et Scientia Vol. 7 (2) Julio - diciembre 2018. 
En el mundo, las ciencias forenses vienen logrando un alto nivel de desarrollo al que indudablemente la odontología viene contribuyendo ((SISIB), 2014). Por eso es necesario desarrollar en el alumno el conocimiento general médico-legal en sus áreas judicial, social y profesional, así como preparar para resolver los problemas odontológico-legales de cualquier nivel de complejidad. Fomentar el interés del alumno en la investigación, la docencia y todo lo que contribuya al progreso científico de la Odontología Legal y forense, su propia actualización y perfeccionamiento.

\section{OBJETIVOS}

a) Identificar los componentes de la motivación y actitud por el aprendizaje de la Odontología Legal y Forense de los estudiantes.

b) Analizar el nivel de consistencia y confiabilidad del instrumento creado para la medición de la motivación y actitud hacia el aprendizaje de la Odontología Legal y Forense.

\section{METODOLOGÍA}

Estudio, analítico, prospectivo, trasversal cuasi experimental. Estuvo conformado por los alumnos que cursaron odontología forense del IX ciclo de la escuela de odontología de la Universidad Privada de Tacna de los años 2016 y 2017. De un total de 52 estudiantes se logró estudiar a 46. Grupo Control (Piloto): Alumnos de odontología del IX ciclo. La medición de las dimensiones de la motivación fue mediante un test de motivación que comprendió la motivación integradora, motivación instrumental, motivación intrínseca y motivación extrínseca. Para la medición de cada ítem se usó los indicadores: Mucho, Bastante, Regular, Poco y Nada. Para obtener las escalas de la motivación general se sumó el total de ítems, luego se calculó la media y varianza. Se utilizó la Escala de intervalo (Baremos). La medición de la actitud hacia el curso de odontología legal y forense se hizo a través de tres componentes: Componente afectivo, componente Cognitivo y componente Conductual. La actitud general se sumó el total de ítems, luego se calculó la media y varianza dando las categorías de Actitud Positiva (>94,4), Actitud Indiferente $(86,2-94.4)$ y Actitud Negativa $(<86,2)$. Se usó la escala de intervalo (Baremos). La técnica de recolección fue personalizada en el grupo experimental, donde se vigiló Verdad, Triangulación, Triangulación de fuentes y Aplicabilidad, Consistencia y Neutralidad. Para diseñar el instrumento se revisa los modelos propuestos en lengua inglesa de Selvajothi (Selvajothi, Lavanya, Joshua, Rao, \& Ranganathan, 2014). Igualmente se revisó el estudio de Hannah (Hannah et al., 2017) quien evaluó el conocimiento, la actitud y la práctica de la odontología forense entre los estudiantes de pregrado en odontología.

\section{RESULTADOS}

En la tabla 1 se muestra el consolidado de los puntajes obtenidos de las fichas de evaluación por seis expertos, logrando obtener un total de 964 puntos.

Veritas et Scientia Vol. 7 (2) Julio - diciembre 2018. 


\section{Tabla 01}

Resultados de la validación por expertos

\begin{tabular}{lllll}
\hline Expertos & Pertinente & Relevante & Comprensivo & Total \\
\hline Experto 1 & 54 & 54 & 54 & 162 \\
\hline Experto 2 & 54 & 52 & 54 & 160 \\
\hline Experto 3 & 56 & 56 & 54 & 166 \\
\hline Experto 4 & 54 & 53 & 51 & 158 \\
\hline Experto 5 & 52 & 51 & 52 & 155 \\
\hline Experto 6 & 54 & 54 & 55 & 163 \\
\hline Puntaje Total & & & 964 \\
\hline
\end{tabular}

El instrumento fue sometido a una prueba piloto a un total de 46 estudiantes de pregrado de noveno ciclo de estudios de la carrera profesional de odontología. Se evidenció un alpha de Cronbach de 0,77 lo que significa un $77 \%$ de confiabilidad por tanto válido para aplicarse.

Se calculó el alpha para cada factor de construcción racional, de forma que hemos calculado la consistencia interna en cada factor. Los resultados son resumidos en la tabla 2. Se eliminaron los ítems que obtuvieron bajos puntajes (M7, M11, M12, M13, M14, M15, M16, M17, M19, M20 Y M24).

El 47,8\% mostró baja motivación en la dimensión integradora y el 32,6\% motivación media, el $43,5 \%$ muestra baja motivación en la dimensión instrumental, el $30,4 \%$ presenta baja motivación intrínseca y el $28,3 \%$ motivación media, luego en la dimensión extrínseca el 39,1\% mostró alta motivación.

La segunda parte del instrumento de refiere a la actitud que el alumno muestra frente al estudio del curso de odontología legal y forense, el cual fue aplicado al mismo tiempo que la primera parte que corresponde a la motivación. El test de actitud consideró en un primer momento 28 ítems. El test en general mostraba Alfa Cronbach de 0.86. Los ítems con bajos puntajes, pero que no aportaban a aumentar la confiabilidad fueron A8, A11, A12, A18, A24, A26 Y A 27; así que se decidió conservarlos (Tabla 03).

Respecto a actitud, el $47,8 \%$ se mostró con una actitud positiva en el plano afectivo, el $50 \%$ tuvo actitud positiva en la dimensión cognitiva y un $47,8 \%$ tiene una actitud conductual positiva. 
Tabla 02

Consistencia interna corregida del test de test de motivación

\begin{tabular}{|c|c|c|c|c|c|c|}
\hline \multicolumn{7}{|c|}{ Estadísticos total-elemento } \\
\hline ítems & $\begin{array}{l}\text { Ítems } \\
\text { Antes }\end{array}$ & Ítems & $\begin{array}{c}\text { Media de } \\
\text { la escala si } \\
\text { se elimina } \\
\text { el } \\
\text { elemento }\end{array}$ & $\begin{array}{c}\text { Varianza } \\
\text { de la } \\
\text { escala si } \\
\text { se elimina } \\
\text { el } \\
\text { elemento } \\
\end{array}$ & $\begin{array}{c}\text { Correlación } \\
\text { elemento- } \\
\text { total } \\
\text { corregida }\end{array}$ & $\begin{array}{l}\text { Alfa de } \\
\text { Cronbach } \\
\text { si se } \\
\text { elimina el } \\
\text { elemento }\end{array}$ \\
\hline M1 & M1 & $\begin{array}{l}\text { Estudiaría/ estudio el curso porque me } \\
\text { gusta la carrera }\end{array}$ & 72.543 & 54.120 & .547 & .749 \\
\hline M2 & M2 & $\begin{array}{l}\text { Estudiaría/ estudio el curso porque me } \\
\text { gustaría tener la experiencia }\end{array}$ & 72.522 & 55.766 & .448 & .756 \\
\hline M3 & M3 & $\begin{array}{l}\text { Estudiaría/ estudio el curso porque lo } \\
\text { necesito/necesitaré en } \mathrm{mi} \text { carrera } \\
\text { profesional. }\end{array}$ & 72.630 & 54.194 & .471 & .752 \\
\hline M4 & M4 & $\begin{array}{l}\text { Estudiaría/ estudio el curso porque me } \\
\text { gusta aprender cosas nuevas. }\end{array}$ & 72.435 & 54.385 & .578 & .749 \\
\hline M5 & M5 & $\begin{array}{l}\text { Estudiaría/ estudio el curso porque lo } \\
\text { necesito para mis estudios. }\end{array}$ & 72.587 & 55.092 & .451 & .754 \\
\hline M6 & M6 & $\begin{array}{l}\text { Estudiaría/ estudio el curso porque me } \\
\text { permite participar mejor en actividades } \\
\text { de investigación }\end{array}$ & 72.696 & 55.994 & .381 & .758 \\
\hline M7 & M7 & $\begin{array}{l}\text { Estudiaría/ estudio el curso porque para } \\
\text { mí es interesante aprender }\end{array}$ & 72.543 & 57.498 & .292 & .764 \\
\hline M8 & M8 & $\begin{array}{l}\text { Estudiaría/ estudio el curso porque me } \\
\text { permitirá ser una persona mejor } \\
\text { instruida/preparada. }\end{array}$ & 72.413 & 56.381 & .356 & .760 \\
\hline M9 & M9 & $\begin{array}{l}\text { Estudiaría/ estudio el curso porque me } \\
\text { permite comprender y apreciar mejor la } \\
\text { odontología y su utilidad }\end{array}$ & 72.304 & 55.461 & .467 & .755 \\
\hline M10 & M10 & $\begin{array}{l}\text { Estudiaría/ estudio el curso porque en } \\
\text { general es gratificante aprender. }\end{array}$ & 72.391 & 55.843 & .344 & .760 \\
\hline M11 & M11 & $\begin{array}{l}\text { Estudiaría/ estudio el curso porque me } \\
\text { sirve para interactuar con otros } \\
\text { profesionales de la salud }\end{array}$ & 72.500 & 57.411 & .261 & .765 \\
\hline M12 & M12 & Mi profesor/a es una persona agradable. & 72.196 & 59.316 & .051 & .775 \\
\hline M13 & M16 & $\begin{array}{l}\text { Mi profesor/a no nos da deberes } \\
\text { adecuados a lo que hemos aprendido en } \\
\text { clase. }\end{array}$ & 73.109 & 55.166 & .242 & .769 \\
\hline M14 & M17 & Mi profesor/a no es organizado/a. & 74.087 & 54.570 & .221 & .773 \\
\hline M15 & M18 & $\begin{array}{l}\text { Para mí no es tan importante el curso } \\
\text { respecto otros }\end{array}$ & 73.848 & 52.310 & .356 & .760 \\
\hline M16 & M19 & $\begin{array}{l}\text { Estudiar el curso es una experiencia que } \\
\text { disfruto/ agradable. }\end{array}$ & 72.696 & 56.350 & .326 & .761 \\
\hline M17 & M20 & $\begin{array}{l}\text { No es realmente necesario estudiar este } \\
\text { curso }\end{array}$ & 73.978 & 55.311 & .214 & .772 \\
\hline M18 & M21 & $\begin{array}{l}\text { Me gustaría aprender varias } \\
\text { experiencias respecto a este curso }\end{array}$ & 72.630 & 55.838 & .380 & .758 \\
\hline M19 & M22 & Prefiero leer la información extranjera & 73.435 & 54.162 & .491 & .751 \\
\hline M20 & M23 & $\begin{array}{l}\text { No creo que sea necesario aprender } \\
\text { este curso en la universidad }\end{array}$ & 73.870 & 52.827 & .299 & .767 \\
\hline M21 & M24 & Debería ser optativo. & 73.891 & 55.432 & .223 & .770 \\
\hline
\end{tabular}

Veritas et Scientia Vol. 7 (2) Julio - diciembre 2018. 
Tabla 03

Consistencia internadel test de actitud hacia el curso.

\begin{tabular}{|c|c|c|c|c|c|c|}
\hline & \multicolumn{6}{|c|}{ Estadísticos total-elemento } \\
\hline $\begin{array}{l}\text { Ítems } \\
\text { ahora }\end{array}$ & $\begin{array}{l}\text { ítems } \\
\text { antes }\end{array}$ & ÍTEMS ACTITUD & $\begin{array}{c}\text { Media de la } \\
\text { escala si se } \\
\text { elimina el } \\
\text { elemento }\end{array}$ & $\begin{array}{c}\text { Varianza de la } \\
\text { escala si se } \\
\text { elimina el } \\
\text { elemento }\end{array}$ & $\begin{array}{l}\text { Correlación } \\
\text { elemento- } \\
\text { total } \\
\text { corregida }\end{array}$ & $\begin{array}{c}\text { Alfa de } \\
\text { Cronbach si } \\
\text { se elimina el } \\
\text { elemento }\end{array}$ \\
\hline $\mathrm{A} 1$ & $\mathrm{~A} 1$ & $\begin{array}{l}\text { La odontología legal y forense es parte de la medicina } \\
\text { forense }\end{array}$ & 92.935 & 76.862 & .540 & .847 \\
\hline $\mathrm{A} 2$ & A2 & $\begin{array}{l}\text { La odontología legal y forense juega un rol importante } \\
\text { en el análisis de la criminalística }\end{array}$ & 92.891 & 78.677 & .485 & .849 \\
\hline A3 & A3 & $\begin{array}{l}\text { El odontólogo puede ser un experto de apoyo en los } \\
\text { casos de problemas legales en lo civil y en los } \\
\text { crímenes }\end{array}$ & 93.043 & 75.154 & .657 & .842 \\
\hline A4 & A4 & $\begin{array}{l}\text { Estoy consciente que existe una rama en odontología } \\
\text { Ilamada odontología forense }\end{array}$ & 93.087 & 76.570 & .637 & .844 \\
\hline A5 & A5 & $\begin{array}{l}\text { Entiendo a la "odontología forense" como parte de mi } \\
\text { plan de estudios }\end{array}$ & 93.304 & 75.683 & .602 & .844 \\
\hline A6 & A6 & $\begin{array}{l}\text { Estoy consciente de que un dentista puede testificar } \\
\text { como un testigo experto en el tribunal para presentar } \\
\text { evidencia dental forense (archivos dentales) }\end{array}$ & 93.152 & 78.932 & .555 & .848 \\
\hline A7 & A7 & $\begin{array}{l}\text { Creo que la odontología forense juega un papel } \\
\text { importante en los desastres masivo }\end{array}$ & 93.043 & 74.709 & .688 & .841 \\
\hline A8 & A8 & $\begin{array}{l}\text { Cree usted que su nivel de conocimiento / } \\
\text { conocimientos sobre la odontología forense es } \\
\text { adecuado }\end{array}$ & 93.391 & 80.910 & .353 & .853 \\
\hline A9 & A9 & $\begin{array}{l}\text { Estoy interesado en un entrenamiento formal en } \\
\text { odontología forense }\end{array}$ & 93.478 & 77.855 & .542 & .847 \\
\hline A10 & A10 & $\begin{array}{l}\text { Creo que hay posibilidades del alcance de la } \\
\text { odontología forense como una profesión }\end{array}$ & 93.152 & 78.443 & .600 & .847 \\
\hline A11 & A11 & Leería revistas relacionadas con la odontología forense & 93.457 & 80.165 & .370 & .853 \\
\hline A12 & A12 & $\begin{array}{l}\text { Creo que la Odontología Forense es un tema } \\
\text { importante }\end{array}$ & 93.152 & 80.621 & .324 & .854 \\
\hline A13 & A13 & $\begin{array}{l}\text { Soy consciente de la importancia de mantener registros } \\
\text { dentales para identificar a fallecidos y sospechosos de } \\
\text { delitos }\end{array}$ & 93.130 & 78.694 & .566 & .847 \\
\hline $\mathrm{A} 14$ & $\mathrm{~A} 14$ & Los hisopos de saliva actúan como fuente de ADN & 93.174 & 78.858 & .512 & .849 \\
\hline A15 & A15 & $\begin{array}{l}\text { La odontología forense es confiable solamente si las } \\
\text { evidencias se recogen dentro de un período de tiempo } \\
\text { del crimen }\end{array}$ & 93.522 & 79.455 & .346 & .854 \\
\hline A16 & A16 & $\begin{array}{l}\text { Podría afirmar que las ideas moleculares de La saliva } \\
\text { puede usarse para resolver cuestiones de } \\
\text { controversias }\end{array}$ & 93.478 & 81.677 & .264 & .856 \\
\hline A17 & A17 & $\begin{array}{l}\text { La odontología forense la percibo amena y estimulante } \\
\text { para mí. }\end{array}$ & 93.500 & 78.833 & .481 & .849 \\
\hline A18 & A18 & La odontología forense es un área valiosa y necesaria & 93.109 & 81.299 & .378 & .853 \\
\hline A19 & A19 & $\begin{array}{l}\text { Pienso que podría estudiar a profundidad la } \\
\text { odontología forense }\end{array}$ & 93.413 & 79.892 & .407 & .852 \\
\hline A20 & A21 & $\begin{array}{l}\text { Siempre dejo en último lugar los asuntos conflictivos y } \\
\text { de investigación porque no me gustan }\end{array}$ & 94.478 & 85.544 & -.041 & .868 \\
\hline A21 & A22 & $\begin{array}{l}\text { La odontología forense y legal me servirán para hacer } \\
\text { estudios de especialización }\end{array}$ & 93.848 & 80.443 & .291 & .856 \\
\hline A22 & A24 & $\begin{array}{l}\text { Siempre soy capaz de controlar mi nerviosismo en los } \\
\text { exámenes a cadáveres }\end{array}$ & 93.848 & 81.554 & .250 & .857 \\
\hline A23 & A25 & $\begin{array}{l}\text { El curso de odontología forense sirve para enseñar a } \\
\text { pensar. }\end{array}$ & 93.630 & 80.016 & .331 & .854 \\
\hline A24 & A26 & $\begin{array}{l}\text { Los términos y artículos legales usados en odontología } \\
\text { nunca me resultan difíciles. }\end{array}$ & 94.152 & 82.265 & .203 & .858 \\
\hline A25 & A27 & $\begin{array}{l}\text { Algunas veces me siento tenso e incómodo en temas } \\
\text { legales y de resolución de conflictos }\end{array}$ & 94.457 & 83.009 & .101 & .864 \\
\hline
\end{tabular}

Test de actitud hacia la odontología legal y forense

Veritas et Scientia Vol. 7 (2) Julio - diciembre 2018. 


\section{DISCUSIÓN}

Se hace necesario medir las habilidades de los estudiantes en el desarrollo de este curso, pero al no encontrar las herramientas necesarias para su medición, es que optamos por la elaboración de un instrumento que realice el sondeo de cómo es que los estudiantes percibieron el curso en las dos únicas promociones que desarrollaron el curso hasta el momento y por haber recientemente ingresado a la malla curricular de la escuela profesional de odontología de la Universidad Privada de Tacna. Galvez et al. en Cuba, realizó un estudio en la ciudad de Eritrea donde se implementaba por primera vez la práctica de la medicina legal, solo con el propósito de informar la frecuencia de actuaciones médico legales realizadas por especialistas cubanos trabajo asistencial que se realizó con los estudiantes de pre y post grado de medicina del periodo 2008 al 2010; donde llegaron a la conclusión que los estudiantes de medicina desarrollaron habilidades en el trabajo médico legal, y que luego fueron constatados durante el periodo de internado. A través del trabajo y los cursos impartidos, quedó demostrado lo necesario que resulta para el médico forense. De igual modo, para nosotros la odontología legal y forense juega un valioso papel sobretodo en la identificación de la persona, cuando así se requiera. De ahí que la elaboración de nuestro instrumento se orienta a identificar la motivación y la actitud que el estudiante de la carrera de odontología presenta con respecto a este nuevo curso para que sirva como herramienta útil para poder tomar decisiones en el modo de trabajo en promociones venideras. Se elaboró un instrumento que muestra la información de nuestras variables de medición en dos partes, la primera parte del instrumento lo hemos denominado test de motivación y la segunda parte se ha denominado Test de actitud. El instrumento ordena los ítems a manera de escala, se calculó la fiabilidad de cada constructo, mejorando la calificación excelente del instrumento para ambas variables, obteniendo de ambas partes lo siguiente. Para la variable motivación se alcanzó una confiabilidad de $77 \%$ y para actitud $85,7 \%$. En Arabia Saudita (Madadin, 2013), se suscitó un estudio para evaluar el conocimiento, actitudes y conciencia de un curso de medicina forense entre estudiantes de medicina de la Universidad de Dammam en el año 2013. Valiéndose del curso de medicina forense evaluó a 143 estudiantes mediante un estudio transversal aplicó un cuestionario de 10 preguntas dirigidas a valorar la conciencia y las actitudes hacia la medicina forense en el pregrado y encontró que los conocimientos eran inadecuados, del cual no pudimos tener alcance al instrumento usado, pero manifestó que las actitudes eran pobres y limitado conocimiento de la importancia que presta el curso de medicina forense entre estudiantes de medicina, es lo más cercano que hemos encontrado respecto al dictado de un curso de medicina legal y forense. En tanto que la mayor parte de los instrumentos que existen para valorar la motivación y la actitud está dirigido a la formación básica, especialmente los cursos de matemáticas y ciencias sociales. Guillermo Vallejo Portuondo, en Santiago de Cuba clasifica la motivación en dos aspectos: una con respecto a la profesión y otra con respecto a la actitud, vinculada al éxito o no en los estudios. Relacionando la motivación y las actitudes con las siguientes formas: motivación profesional como motivación primaria por la carrera y motivación por la profesión como motivación secundaria por la carrera, llegó a la conclusión de que las actitudes influyen en la calidad del aprendizaje, pues las actitudes negativas de diversos tipos hacia el estudio, conllevan a que el alumno no logre aprender los contenidos con la calidad deseada. No se ha encontrado otro estudio sobre motivación y actitudes orientadas a la odontología forense, pero en el Perú Díaz et. al. Buscó determinar los conocimientos, actitudes y prácticas en investigación de los estudiantes de Medicina del Perú. Donde la tercera parte del instrumento se refería a las actitudes del alumno

Veritas et Scientia Vol. 7 (2) Julio - diciembre 2018. 
frente a la investigación siendo 18 ítems los que fueron elaborados en escala Likert se consideró buena actitud al puntaje total igual o mayor a 63 puntos. $Y$ encontraron que los estudiantes tenían actitudes positivas para investigar y no era necesario ser considerado superdotado que bastaba con la intención de implicarse en un trabajo de investigación (80\%). (Díaz Vélez, Manrique González, Galán Rodas, \& Apolaya Segura, 2008).

Se recomienda al personal docente universitario, aplicar instrumentos que midan la visión del estudiante con respecto a la asignatura que están desarrollando, con el objeto que en lo sucesivo mejorar con estrategias metodológicas y didácticas actitudes más positivas en el aprendizaje de un curso. Implementar en el proceso de enseñanza estrategias específicas que ayuden a mejorar la motivación y la actitud en los estudiantes.

\section{REFERENCIAS BIBLIOGRÁFICAS}

Barboza Alvarado, María E. (1987) Algunas teorías pedagógicas que le han dado sustento a la metodología de enseñanza - aprendizaje serie: textos pedagógicos. Universidad de Costa rica Escuela de Trabajo social. Pp 63.

Carrillo Esper, R., Contreras Carreto, N. A., Remolina Schlig, M., \& Ramírez Rosillo, F. J. (2007). Comunicando malas noticias en la Unidad de Terapia Intensiva "Primum non nocere". Rev. de la Asoc. Mex. de Med. Crítica y Terapia Intensiva, XXI(4), 194-199.

Díaz Vélez, C., Manrique González, L. M., Galán Rodas, E., \& Apolaya Segura, M. (2008). Conocimientos, actitudes y prácticas en investigación de los estudiantes de pregrado de facultades de medicina del Perú. Acta Médica Peruana, 25(1), 9-15.

INCIFO. (2017). Odontología forense. Instituto de Ciencias Forenses Poder Judicial de la Ciudad de México http://incifocdmx.gob.mx/odontologia-forense/forme de Zotero [Institucional]. Recuperado 27 de agosto de 2017, a partir de zotero://report/items/0_85JJVVJA/html/report.html

Kusurkar, R. A., Ten Cate, T. J., Vos, C. M. P., Westers, P., \& Croiset, G. (2013). How motivation affects academic performance: a structural equation modelling analysis. Advances in Health Sciences Education, 18(1), 57-69. https://doi.org/10.1007/s10459-012-9354-3

Madadin, M. S. (2013). Assessment of knowledge about, attitudes toward, and awareness of a forensic medicine course among medical students at the University of Dammam. Journal of Forensic and Legal Medicine, 20(8), 1108-1111. https://doi.org/10.1016/j.jflm.2013.10.003

Minera Reyna, L. E. (2009). El papel de la motivación y las actitudes en el aprendizaje de ELE en un contexto de enseñanza formal para adultos alemanes. ISSN 1699-6569. Revista lingüística, 6(2). Recuperado a partir de http://www.nebrija.com/revista-linguistica/elpapel-de-la-motivacion-y-las-actitudes-en-el-aprendizaje-de-ele-en-un-contexto-deense\%C3\%B1anza-formal-para-adultos-alemanes 
Ministerio Público. Reglamento de Organización y funciones del Instituto de Medicina Legal. Resolución Administrativa del titular del Ministerio Público N³84-98-SE-TP-CEMP. Perú (1998). Recuperado a partir de http://sistemas.amag.edu.pe/publicaciones/administ_justicia/gestion_fiscal/99114.pdf

Ministerio Público. (2017). Instituto de medicina legal y ciencias forenses. [Fiscalía de la Nación]. Recuperado 27 de agosto de 2017, a partir de http://www.mpfn.gob.pe/iml/quienes_somos/

Quintero, J. (2012). Abraham Maslow y su teoría de la motivación humana [psicopedagogiaaprendizajeuc]. Recuperado 1 de septiembre de 2017, a partir de https://psicopedagogiaaprendizajeuc.wordpress.com/2012/06/29/abraham-maslow-ysu-teoria-de-la-motivacion-humana/

Roberts, L. D., Howell, J. A., Seaman, K., \& Gibson, D. C. (2016). Student Attitudes toward Learning Analytics in Higher Education: "The Fitbit Version of the Learning World". Frontiers in Psychology, 7. https://doi.org/10.3389/fpsyg.2016.01959

Rodríguez Jorge, R. R., \& Loy Vera, B. (2016). Bases teóricas de las ciencias forenses contemporáneas y las competencias interdisciplinarias profesionales. Medicentro Electrónica, 20(1), 3-10.

Sandoval-Pineda, A. (2011). Attitude, motivation and English language learning in a Mexican college context. Recuperado a partir de http://arizona.openrepository.com/arizona/handle/10150/145743

(SISIB). (2014). Programa Profesional de Especialista en odontología legal y forense. Universidad de Chile Sistema de Servicios de Información y Bibliotecas [Text]. Recuperado 1 de septiembre de 2017, a partir de http://www.uchile.cl/postgrados/108049/odontologia-legal-y-forense

Ubillos, S., Mayordomo, S., \& Páez, D. (2015). Psicología Social, Cultura y Educación, Capítulo X: Actitudes: definición y medición Componetes de la actitud. modelo de accion razonada y acción planificada. Recuperado a partir de www.ehu.eus/documents/1463215/1504276/Capitulo+X.pdf 